\title{
The Non-Local Theory Solution of Orthotropic Composite Materials on the Stress Field near the Crack Tips*
}

\author{
Yin-Huan YANG** \\ ${ }^{*}$ NO.1 Xuehai Street, Songbei District, School of Civil Engineering and Refrigeration Engineering, \\ Harbin University of Commerce, Harbin, 150028, P. R. China \\ E-mail: yinhuanyang@yahoo.com.cn
}

\begin{abstract}
The effect of the lattice parameter of orthotropic composite materials on the stress fields near crack tips was investigated by means of non-local theory. By use of the Fourier transform, the problem can be solved with the help of a pair of dual integral equations, in which the unknown variable is the displacement along the crack surfaces. To solve the dual integral equations, the displacement along the crack surfaces was directly expanded as a series of Jacobi polynomials. The solution can be obtained by means of the Schmidt method. Different from the classical solutions, that the present soluction exhibits no stress singularity at the crack tips, i.e. the stress field near the crack tips is finite. The effect of the lattice parameter on the stress field near the crack tips was displayed graphically.
\end{abstract}

Key words: the Lattice Parameter, Crack, Non-Local Theory, Orthotropic Composite Materials

\section{Introduction}

In recent years, composite materials are being used in a wide range of engineering field. The fracture of composite materials is induced mainly from propagation of a crack. Hence, the fracture problem of orthotropic materials is very important from the view point of the strength and stress analysis of cracks have been treated in many papers [1-3]. However, it was found that most available solutions in the literature [1-3] exhibit stress singularities at the crack tips, which is not reasonable according to the physical nature of fracture. In fact, the stresses at the crack tips are finite. As a result of this, beginning with Griffith, all fracture criteria in practice today are based on other considerations, such as energy, and the J-integral [4].

In contrast to the local approach of zero-range internal interactions, the modern non-local continuum mechanics, originated and developed during the last four decades, postulates that the local state at a point of a body is influenced by the action of all particles of the body. This theory was constructed primarily by Edelen [5], Eringen [6], Green and Rivlin [7]. In reference [8], the basic theory of non-local elasticity was described with emphasis on the difference between the non-local theory and classical continuum mechanics. To overcome the stress singularity at the crack tips in the classical elastic theory, Eringen [9-11] used non-local theory to discuss the stress near the tip of a sharp line crack in an isotropic elastic plate subject to uniform tension, shear and anti-plane shear loading. His solutions did not contain any stress singularities at the crack tips. This enables us to employ the maximum stress criterion to predict fracture in a more natural way. Recently, some fracture problems [12-14] in isotropic elastic materials or piezoelectric materials have been studied by using 
non-local theory and the Schmidt method [15]. To our knowledge, the behavior of a Griffith crack in orthotropic composite materials subjected to a uniform tension has not been studied by means of non-local theory in any open literature. It is with this in mind that we offer the present work.

\section{Basic equation of non-local theory}

The basic equations for a two-dimensional plane of homogeneous, orthotropic, non-local elastic material, with vanishing body force are $[3,11]$

$$
\begin{gathered}
\frac{\partial \tau_{x}}{\partial x}+\frac{\partial \tau_{x y}}{\partial y}=0, \frac{\partial \tau_{x y}}{\partial x}+\frac{\partial \tau_{y}}{\partial y}=0 \\
\tau_{x}(x, y)=\int_{-\infty}^{\infty} \int_{-\infty}^{\infty}\left[A_{11}^{*}\left(\left|x^{\prime}-x\right|,\left|y^{\prime}-y\right|\right) \frac{\partial u\left(x^{\prime}, y^{\prime}\right)}{\partial x^{\prime}}+A_{12}^{*}\left(\left|x^{\prime}-x\right|,\left|y^{\prime}-y\right|\right) \frac{\partial v\left(x^{\prime}, y^{\prime}\right)}{\partial y^{\prime}}\right] d x^{\prime} d y^{\prime} \\
\tau_{y}(x, y)=\int_{-\infty}^{\infty} \int_{-\infty}^{\infty}\left[A_{12}^{*}\left(\left|x^{\prime}-x\right|,\left|y^{\prime}-y\right|\right) \frac{\partial u\left(x^{\prime}, y^{\prime}\right)}{\partial x^{\prime}}+A_{22}^{*}\left(\left|x^{\prime}-x\right|,\left|y^{\prime}-y\right|\right) \frac{\partial v\left(x^{\prime}, y^{\prime}\right)}{\partial y^{\prime}}\right] d x^{\prime} d y^{\prime} \\
\tau_{x y}(x, y)=\int_{-\infty}^{\infty} \int_{-\infty}^{\infty} A_{66}^{*}\left(\left|x^{\prime}-x\right|,\left|y^{\prime}-y\right|\right)\left[\frac{\partial u\left(x^{\prime}, y^{\prime}\right)}{\partial y^{\prime}}+\frac{\partial v\left(x^{\prime}, y^{\prime}\right)}{\partial x^{\prime}}\right] d x^{\prime} d y^{\prime}
\end{gathered}
$$

where $u(x, y)$ and $v(x, y)$ are the displacements in $x$ and $y$ directions, respectively. The only difference from the classical elasticity is in the stress constitutive equations (2)-(4) in which the stresses $\tau_{x}, \tau_{y}$ and $\tau_{x y}$, at a point $(x, y)$ depend on the strains $\frac{\partial u(x, y)}{\partial x}$, $\frac{\partial v(x, y)}{\partial y}, \frac{\partial u(x, y)}{\partial y}$ and $\frac{\partial v(x, y)}{\partial x}$, at all points of the body. For a homogeneous and orthotropic composite material plane there exit only four materials parameters $A_{11}^{*}\left(\left|x^{\prime}-x\right|,\left|y^{\prime}-y\right|\right), \quad A_{12}^{*}\left(\left|x^{\prime}-x\right|,\left|y^{\prime}-y\right|\right), \quad A_{22}^{*}\left(\left|x^{\prime}-x\right|,\left|y^{\prime}-y\right|\right) \quad$ and $A_{66}^{*}\left(\left|x^{\prime}-x\right|,\left|y^{\prime}-y\right|\right) \quad$ which are functions of the distance $d=\sqrt{\left(x^{\prime}-x\right)^{2}+\left(y^{\prime}-y\right)^{2}}$ between the point of interest and an arbitrary point in the body. As discussed in [16], the forms of $A_{11}^{*}\left(\left|x^{\prime}-x\right|,\left|y^{\prime}-y\right|\right)$, $A_{12}^{*}\left(\left|x^{\prime}-x\right|,\left|y^{\prime}-y\right|\right), \quad A_{22}^{*}\left(\left|x^{\prime}-x\right|,\left|y^{\prime}-y\right|\right)$ and $A_{66}^{*}\left(\left|x^{\prime}-x\right|,\left|y^{\prime}-y\right|\right)$ can be assumed as follows:

$$
\begin{aligned}
& {\left[A_{11}^{*}, \quad A_{12}^{*}, \quad A_{22}^{*}, \quad A_{66}^{*}\right]=\left[A_{11}, \quad A_{12}, \quad A_{22}, \quad A_{66}\right] \alpha\left(\left|x^{\prime}-x\right|,\left|y^{\prime}-y\right|\right)} \\
& \alpha\left(\left|x^{\prime}-x\right|,\left|y^{\prime}-y\right|\right)=\alpha_{0} \exp \left\{-(\beta / a)^{2}\left[\left(x^{\prime}-x\right)^{2}+\left(y^{\prime}-y\right)^{2}\right]\right\}
\end{aligned}
$$

where $\alpha\left(\left|x^{\prime}-x\right|,\left|y^{\prime}-y\right|\right)$ is known as the influential function. $\beta$ and $a$ are material constants. Normally $a$ is known as the characteristic length of a material which may be selected according to the range and sensitivity of the physical phenomena to be investigated. For perfect crystals, a may be taken as the lattice parameter. For granular materials, $a$ may be considered to be the average granular distance and for fiber composites, the fiber distance, etc. The material constant $\beta$ may be determined experimentally. $A_{11}, A_{12}, A_{22}$ and $A_{66}$ are elastic moduli of the orthotropic material. $\alpha_{0}$ is determined by the normalization

$$
\int_{-\infty}^{\infty} \int_{-\infty}^{\infty} \alpha\left(\left|x^{\prime}-x\right|,\left|y^{\prime}-y\right|\right) d x^{\prime} d y^{\prime}=1
$$


In the present work we employ non-local elastic moduli given by Eq. (5) and Eq. (6). Substituting Eq. (5) and Eq. (6) into Eq. (7), we can obtained, in a two-dimensional space,

$$
\alpha_{0}=\frac{1}{\pi}(\beta / a)^{2}
$$

Substitution Eqs. (2)-(6) into Eq. (1) and using the Green-Gauss theorem, we have

$$
\begin{gathered}
\int_{-\infty}^{\infty} \int_{-\infty}^{\infty} \alpha\left(\left|x^{\prime}-x\right|,\left|y^{\prime}-y\right|\right)\left[A_{11} \frac{\partial^{2} u\left(x^{\prime}, y^{\prime}\right)}{\partial x^{\prime 2}}+A_{66} \frac{\partial^{2} u\left(x^{\prime}, y^{\prime}\right)}{\partial y^{\prime 2}}+\left(A_{12}+A_{66}\right) \frac{\partial^{2} v\left(x^{\prime}, y^{\prime}\right)}{\partial x^{\prime} \partial y^{\prime}}\right] d x^{\prime} d y^{\prime} \\
-\int_{-l}^{l} \alpha\left(\left|x^{\prime}-x\right|,\left|y^{\prime}-y\right|\right)\left[\sigma_{x y}\left(x^{\prime}, 0^{+}\right)-\sigma_{x y}\left(x^{\prime}, 0^{-}\right)\right] d x^{\prime}=0 \\
\int_{-\infty}^{\infty} \int_{-\infty}^{\infty} \alpha\left(\left|x^{\prime}-x\right|,\left|y^{\prime}-y\right|\right)\left[A_{66} \frac{\partial^{2} v\left(x^{\prime}, y^{\prime}\right)}{\partial x^{\prime 2}}+A_{22} \frac{\partial^{2} v\left(x^{\prime}, y^{\prime}\right)}{\partial y^{\prime 2}}+\left(A_{12}+A_{66}\right) \frac{\partial^{2} u\left(x^{\prime}, y^{\prime}\right)}{\partial x^{\prime} \partial y^{\prime}}\right] d x^{\prime} d y^{\prime} \\
-\int_{-l}^{l} \alpha\left(\left|x^{\prime}-x\right|,\left|y^{\prime}-y\right|\right)\left[\sigma_{y}\left(x^{\prime}, 0^{+}\right)-\sigma_{y}\left(x^{\prime}, 0^{-}\right)\right] d x^{\prime}=0
\end{gathered}
$$

where

$$
\left\{\begin{array}{l}
\sigma_{x}(x, y)=\mu\left[c_{11} \frac{\partial u(x, y)}{\partial x}+c_{12} \frac{\partial v(x, y)}{\partial y}\right] \\
\sigma_{y}(x, y)=\mu\left[c_{12} \frac{\partial u\left(x^{\prime}, y^{\prime}\right)}{\partial x^{\prime}}+c_{22} \frac{\partial v(x, y)}{\partial y}\right] \\
\sigma_{x y}(x, y)=\mu\left(\frac{\partial u(x, y)}{\partial y}+\frac{\partial v(x, y)}{\partial x}\right)
\end{array}\right.
$$

where $c_{11}=A_{11} / A_{66}, \quad c_{12}=A_{12} / A_{66}, \quad c_{22}=A_{22} / A_{66}, \mu=A_{66} . l$ is the half-length of the crack which will be discussed shortly. The expressions of Eq. (11) are the classical Hooke's law. It is worth noting that the surface integrals in Eq.(9) and Eq. (10) may be dropped since the displacement field vanishes at infinity..

As discussed in [9], it can be obtained that $\sigma_{x y}\left(x, 0^{+}\right)-\sigma_{x y}\left(x, 0^{-}\right)=0$ and $\sigma_{y}\left(x, 0^{+}\right)-\sigma_{y}\left(x, 0^{-}\right)=0$. Therefore it can be shown that the general solutions of Eq. (9) and Eq. (10) are identical to those of the following two differential equations almost everywhere over the entire plane:

$$
\begin{aligned}
& c_{11} \frac{\partial^{2} u}{\partial x^{2}}+\frac{\partial^{2} u}{\partial y^{2}}+\left(1+c_{12}\right) \frac{\partial^{2} v}{\partial x \partial y}=0 \\
& c_{22} \frac{\partial^{2} v}{\partial y^{2}}+\frac{\partial^{2} v}{\partial x^{2}}+\left(1+c_{12}\right) \frac{\partial^{2} u}{\partial x \partial y}=0
\end{aligned}
$$

The non-dimensional parameters $c_{i k}(i, k=1,2)$ involved in the above equations are related to the elastic constants by the relations [3]: $c_{11}=E_{1} /\left[\mu\left(1-v_{12}^{2} E_{2} / E_{1}\right)\right]$, $c_{22}=E_{2} /\left[\mu\left(1-v_{12}^{2} E_{2} / E_{1}\right)\right]=c_{11} E_{2} / E_{1}, c_{12}=v_{12} E_{2} /\left[\mu\left(1-v_{12}^{2} E_{2} / E_{1}\right)\right]=v_{12} c_{22}=v_{21} c_{11}$ for generalized plane stress, and by $c_{11}=E_{1}\left(1-v_{23} v_{32}\right) /(\Delta \mu), c_{22}=E_{2}\left(1-v_{13} v_{31}\right) /(\Delta \mu)$, $c_{12}=E_{1}\left(v_{21}+v_{13} v_{32} E_{2} / E_{1}\right) /(\Delta \mu)=E_{2}\left(v_{12}+v_{23} v_{31} E_{1} / E_{2}\right) /(\Delta \mu)$ 
$\Delta=1-v_{12} v_{21}-v_{23} v_{32}-v_{31} v_{13}-v_{12} v_{23} v_{31}-v_{13} v_{21} v_{32}$ for plane strain. The elastic constants $E_{i}$ and the Poisson's ratio $v_{i k}(i, k=1,2,3)$ satisfy Maxwell's relation $v_{i k} / E_{i}=v_{k i} / E_{k}$. In this paper, we just consider the generalized plane stress case.

\section{The crack model}

It was assumed that there is a crack of length $2 l$ along the $x$-axis in orthotropic elastic materials as shown in Fig.1. It is assumed that a tension stress loading $\tau_{y}=-\tau_{0} \quad\left(\tau_{0}\right.$ is a magnitude of the uniform stress loading) was directly applied on the crack surfaces, which is equivalent to investigating the perturbation fields for a remotely loaded cracked-body through the standard superposition technique in fracture mechanics. As discussed in reference [9], the boundary conditions can be written as following:

$$
\begin{aligned}
& \tau_{y}\left(x, 0^{+}\right)=\tau_{y}\left(x, 0^{-}\right)=-\tau_{0},|x| \leq l \\
& \tau_{x y}\left(x, 0^{+}\right)=\tau_{x y}\left(x, 0^{-}\right)=0,|x| \leq \infty \\
& v\left(x, 0^{+}\right)=v\left(x, 0^{-}\right)=0,|x|>l
\end{aligned}
$$

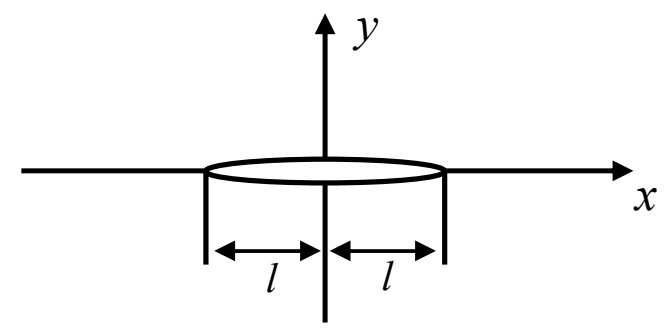

Fig.1. Geometry and coordinate system for the crack

\section{The dual integral equations}

Because of the symmetry, it suffices to consider the problem in the first quadrant: $x \geq 0, y \geq 0$. Consequently, the solution of equations (12)-(13) can be obtained as follows:

$$
\begin{aligned}
& u(x, y)=\frac{2}{\pi} \int_{0}^{\infty}\left[A_{1}(s) e^{-\gamma_{1} s y}+A_{2}(s) e^{-\gamma_{2} s y}\right] \sin (s x) d s, \quad y \geq 0 \\
& v(x, y)=\frac{2}{\pi} \int_{0}^{\infty}\left[\alpha_{1} A_{1}(s) e^{-\gamma_{1} s y}+\alpha_{2} A_{2}(s) e^{-\gamma_{2} s y}\right] \cos (s x) d s, \quad y \geq 0
\end{aligned}
$$

where $\alpha_{1}=\frac{c_{11}-\gamma_{1}^{2}}{\left(1+c_{12}\right) \gamma_{1}}, \quad \alpha_{2}=\frac{c_{11}-\gamma_{2}^{2}}{\left(1+c_{12}\right) \gamma_{2}}, A_{j}(s)(j=1,2)$ are unknown functions to be determined by the boundary conditions. Quantities $r_{j}^{2}(j=1,2)$ are the real and positive roots of the following characteristic equation:

$$
c_{22} \gamma^{4}+\left[c_{12}^{2}+2 c_{12}-c_{11} c_{22}\right] \gamma^{2}+c_{11}=0
$$

Substituting Eq. (17) and Eq. (18) into Eq. (11), we have 


$$
\begin{gathered}
\sigma_{y}(x, y)=\frac{2 \mu}{\pi} \int_{0}^{\infty} s\left[d_{1} A_{1}(s) e^{-\gamma_{1} s y}+d_{2} A_{2}(s) e^{-\gamma_{2} s y}\right] \cos (s x) d s \\
\sigma_{x y}(x, y)=-\frac{2 \mu}{\pi} \int_{0}^{\infty} s\left[d_{3} A_{1}(s) e^{-\gamma_{1} s y}+d_{4} A_{2}(s) e^{-\gamma_{2} s y}\right] \sin (s x) d s
\end{gathered}
$$

where $d_{1}=c_{12}-c_{22} \alpha_{1} \gamma_{1}, d_{2}=c_{12}-c_{22} \alpha_{2} \gamma_{2}, d_{3}=\alpha_{1}+\gamma_{1}, d_{4}=\alpha_{2}+\gamma_{2}$.

According to the boundary condition of Eq. (15), $\sigma_{x y}$ must vanish at $y=0$. Therefore

$$
A_{2}(s)=\frac{d_{3}}{d_{4}} A_{1}(s)
$$

Substituting Eq. (20) and Eq. (21) into Eq. (3) and applying Eq. (5), Eq. (6) and Eq. (22), we have

$$
\begin{aligned}
& \tau_{y}(x, y)=\frac{2 \mu}{\pi} \int_{0}^{\infty} s A_{1}(s) d s \\
& \quad \int_{0}^{\infty}\left(d_{1} e^{-\gamma_{1} s y^{\prime}}+\frac{d_{2} d_{3}}{d_{4}} e^{-\gamma_{2} s y^{\prime}}\right) d y^{\prime} \int_{-\infty}^{\infty}\left[\alpha\left(\left|x^{\prime}-x\right|,\left|y^{\prime}-y\right|\right)+\alpha\left(\left|x^{\prime}-x\right|,\left|y^{\prime}+y\right|\right)\right] \cos \left(s x^{\prime}\right) d x^{\prime}, \quad y \geq 0
\end{aligned}
$$

Substituting for $\alpha$ from Eq. (6), the integrations may be performed with respect to $x^{\prime}$ and $y^{\prime}$, we have

$$
\tau_{y}(x, y)=\frac{\mu}{\pi} \int_{0}^{\infty} s\left[g_{1}(s, y)+g_{2}(s, y)\right] A_{1}(s) \cos (s x) d s
$$

where $g_{1}(s, y)=d_{1}\left\{\left(e^{-\gamma_{1} s y-\frac{s^{2}-\gamma_{1}^{2} s^{2}}{4 p}}\right)\left[1-\Phi\left(\frac{\gamma_{1} s-2 p y}{2 \sqrt{p}}\right)\right]+\left(e^{\gamma_{1} s y-\frac{s^{2}-\gamma_{1}^{2} s^{2}}{4 p}}\right)\left[1-\Phi\left(\frac{\gamma_{1} s+2 p y}{2 \sqrt{p}}\right)\right]\right\}$,

$g_{2}(s, y)=\frac{d_{2} d_{3}}{d_{4}}\left\{\left(e^{-\gamma_{2} s y-\frac{s^{2}-\gamma_{2}^{2} s^{2}}{4 p}}\right)\left[1-\Phi\left(\frac{\gamma_{2} s-2 p y}{2 \sqrt{p}}\right)\right]+\left(e^{\gamma_{2} s y-\frac{s^{2}-\gamma_{2}^{2} s^{2}}{4 p}}\right)\left[1-\Phi\left(\frac{\gamma_{2} s+2 p y}{2 \sqrt{p}}\right)\right]\right\}$,

$\Phi(z)=\frac{2}{\sqrt{\pi}} \int_{0}^{z} \exp \left(-t^{2}\right) d t, \quad p=\left(\frac{\beta}{a}\right)^{2}$.

Then substituting eq. (24) into eqs. (14) and (16) we can have the following dual integral equations about $A_{1}(s)$ :

$$
\begin{aligned}
& \frac{\mu}{\pi} \int_{0}^{\infty} s g(s) A_{1}(s) \cos (s x) d s=-\tau_{0}, 0 \leq x \leq l \\
& \int_{0}^{\infty} A_{1}(s) \cos (s x) d s=0, \quad x>l
\end{aligned}
$$

where $g(s)=g_{1}(s, 0)+g_{2}(s, 0)$. It can be obtained that $\operatorname{Lim}_{a \rightarrow 0} e^{ \pm \gamma_{1} s y-\frac{s^{2}-\gamma_{1}^{2} s^{2}}{4 p}}=1$, $\operatorname{Lim}_{a \rightarrow 0} e^{ \pm \gamma_{2} s y-\frac{s^{2}-\gamma_{2}^{2} s^{2}}{4 p}}=1 \quad, \quad \operatorname{Lim}_{a \rightarrow 0} \Phi\left(\frac{\gamma_{1} s \pm 2 p y}{2 \sqrt{p}}\right)=0, \quad \operatorname{Lim}_{a \rightarrow 0} \Phi\left(\frac{\gamma_{2} s \pm 2 p y}{2 \sqrt{p}}\right)=0 \quad$ when $\quad y=0 \quad$ and $\operatorname{Lim}_{a \rightarrow 0} g(s)=2 d_{1}+\frac{2 d_{2} d_{3}}{d_{4}}$. Therefore Eq. (25) and Eq. (26) will recast the well-known dual integral equations of the classical mechanics when $a \rightarrow 0[19,20]$. The above a pair of dual integral Eq. (25) and Eq. (26) must be solved to determine the unknown function 
$A_{1}(s)$

\section{Solution of the dual integral equations}

The Schmidt method [15] was used to solve the dual integral Eq. (25) and Eq. (26). First, the displacement $v$ along the crack surfaces was represented by the following series:

$$
v(x, 0)=\left\{\begin{array}{l}
\sum_{n=0}^{\infty} a_{n} P_{2 n}^{(1 / 2,1 / 2)}\left(\frac{x}{l}\right)\left(1-\frac{x^{2}}{l^{2}}\right)^{\frac{1}{2}}, \quad 0 \leq x \leq l \\
0, \quad x>l
\end{array}\right.
$$

where $a_{n}$ are unknown coefficients, $P_{n}^{(1 / 2,1 / 2)}(x)$ is a Jacobi polynomial [17]. The Fourier cosine transform of Eq. (27) is [18]

$$
\left(\alpha_{1}+\frac{\alpha_{2} d_{3}}{d_{4}}\right) A_{1}(s)=\bar{v}(s, 0)=\sum_{n=0}^{\infty} a_{n} Q_{n} \frac{1}{S} J_{2 n+1}(s l), \quad Q_{n}=\sqrt{\pi}(-1)^{n} \frac{\Gamma\left(2 n+1+\frac{1}{2}\right)}{(2 n) !}
$$

where $\Gamma(x)$ and $J_{n}(x)$ are the Gamma and Bessel functions, respectively. An overbar indicates the Fourier cosine transform about $x$ throughout the paper.

Substituting the expression of $A_{1}(s)$ as shown in Eq. (28) into Eq. (25) and Eq. (26), it can be shown that Eq. (26) was automatically satisfied. Equation (25) was reduced to the form as follow:

$$
\frac{\mu d_{4}}{\pi\left(\alpha_{1} d_{4}+\alpha_{2} d_{3}\right)} \sum_{n=0}^{\infty} \int_{0}^{\infty} a_{n} Q_{n} g(s) J_{2 n+1}(s l) \cos (s x) d s=-\tau_{0}, 0 \leq x \leq l
$$

Equation (29) can be solved for coefficients $a_{n}$ by the Schmidt method as shown in [13-15, 19, 20]. For brevity, the details are omitted here.

\section{Numerical calculations and discussion}

The coefficients $a_{n}$ are known, so that the entire stress field can be obtained. However, in fracture mechanics, it is important in fracture mechanics to determine the stress $\tau_{y}$ in the vicinity of the crack tips. In present case, $\tau_{y}$ along the crack line can be expressed as follow:

$$
\tau_{y}=\frac{\mu d_{4}}{\pi\left(\alpha_{1} d_{4}+\alpha_{2} d_{3}\right)} \sum_{n=0}^{\infty} \int_{0}^{\infty} a_{n} Q_{n} g(s) J_{2 n+1}(s l) \cos (s x) d s
$$

For the lattice parameter $a \rightarrow 0$ at $x=l$, we have the classical stress singularity. However, so long as the lattice parameter $a \neq 0$, the semi-infinite integration and the series in Eq. (30) are convergent for any variable $x$. Then equation (30) represents a finite stress along $y=0$, and no stress singularity exhibits at the crack tips. In $0<x<l, \tau_{y} /\left(-\tau_{0}\right)$ is very close to unity, and for $x>l, \tau_{y} /\left(-\tau_{0}\right)$ possesses finite values diminishing from a finite value at $x=l$ to zero at $x=\infty$. In all computations, the material constants of Boron epoxy are adopted as $E_{1}=207 G P a, \quad E_{2}=19 G P a, \quad v_{12}=0.21, \quad v_{23}=0.21$ and 
$\mu_{12}=6.4 G P a$. From references $[12,13]$, it can be seen that the Schmidt method is performed satisfactorily if the first ten terms of infinite series to Eq. (29) are retained. The results are plotted in Fig. 2 to Fig. 5. Some conclusions are drawn as follows:

(i) The maximum stresses do not occur at the crack tips, but slightly away from it as shown in Fig. 2 and Fig. 3. This phenomenon has been thoroughly substantiated by Eringen [21]. The maximum stress is finite. The distance between crack tip and the maximum stress point is very small, and it depends on the crack length, the material properties and the lattice parameter. Different from the classical elasticity solution, it is found that no stress singularities are present at the crack tips. Also the present results converge to the classical ones in the regions far away from crack tips. However, $\tau_{y} /\left(-\tau_{0}\right)$ is not close to unity in a very small region near the crack tips, in which it was caused by the computing errors and was hard to avoid as shown in Fig. 3.

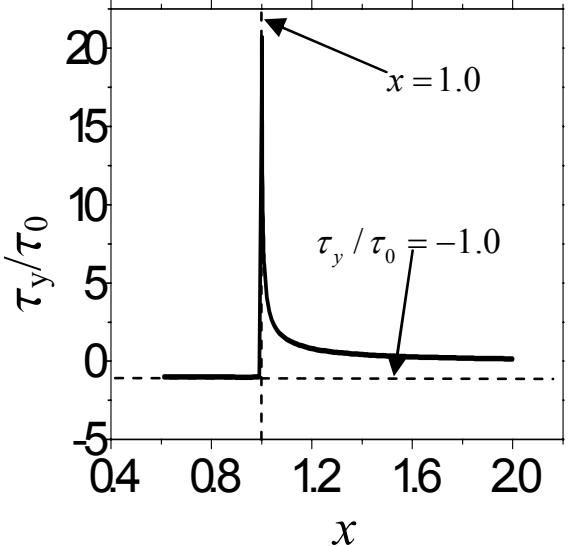

Fig. 2. The stress along the crack line versus $x$ for $l=1.0, a /(\beta l)=0.001$.

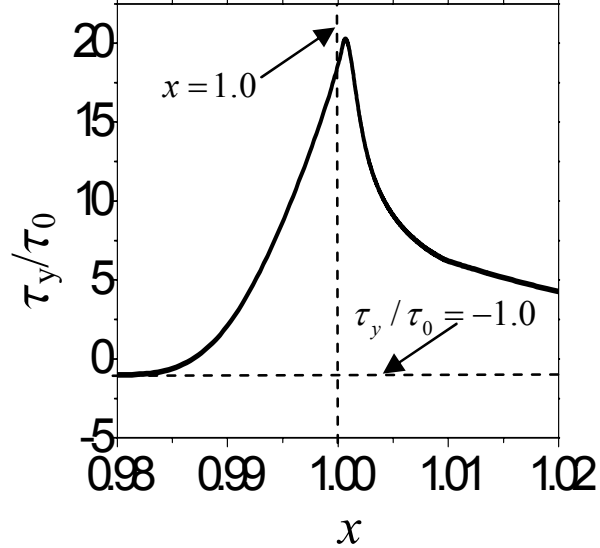

Fig. 3. The locally enlarged graph of figure 2 near the crack tip.

(ii) The stresses at the crack tips become infinite as the lattice parameter $a \rightarrow 0$. This is the classical continuum limit of square root singularity. This can be seen from Eq. (25) and Eq. (26).

(iii) For the $a / \beta=$ constant, viz., the lattice parameter does not change; the value of the stress (at the crack tips) almost linearly increases with increase of the crack length as shown in Fig. 4. This is consistent with experimental results which indicate that smaller cracks are more resistant to fracture than larger cracks. Although the classical fracture mechanics can also reveal this through the concept of stress intensity factor, this difference is not directly distinguished from the singular stress fields around both a smaller and a larger crack. This is one of the major advantages of the non-local fracture mechanics.

(iv) The effect of the lattice parameter, $a$ of orthotropic composite materials on the stress field near the crack tips decreases with increase of the lattice parameter as shown in Fig. 5.

(vi) The stress concentration occurs at crack tip as stating by Eringen [9-11], which is given by

$$
\tau_{y}(l, 0) /\left(-\tau_{0}\right)=c_{0} / \sqrt{a /(2 \beta l)}
$$

where $c_{0}$ represents the stress concentration value at the crack tips. $c_{0}$ is approximately 
equal to 0.394 for Boron epoxy. While for E-glass epoxy, $c_{0}$ is about equal to 0.377 and for Graphite epoxy, $c_{0}$ is about equal to 0.3655 . Therefore we conclude that $c_{0}$ depends on the material properties.

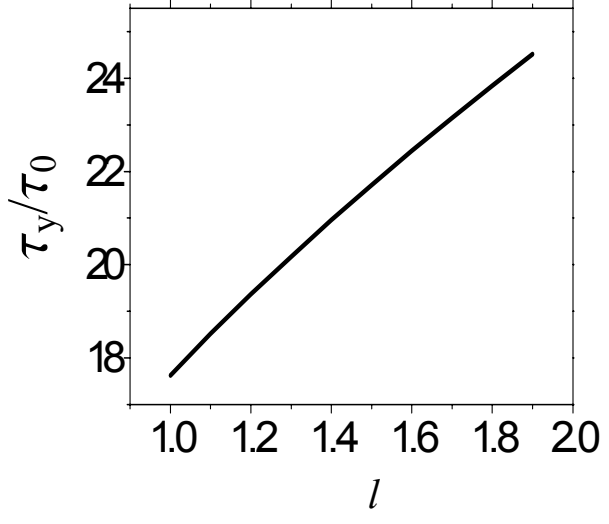

Fig. 4. The stress at the crack tip versus $l$ for $a /(\beta l)=0.001$.

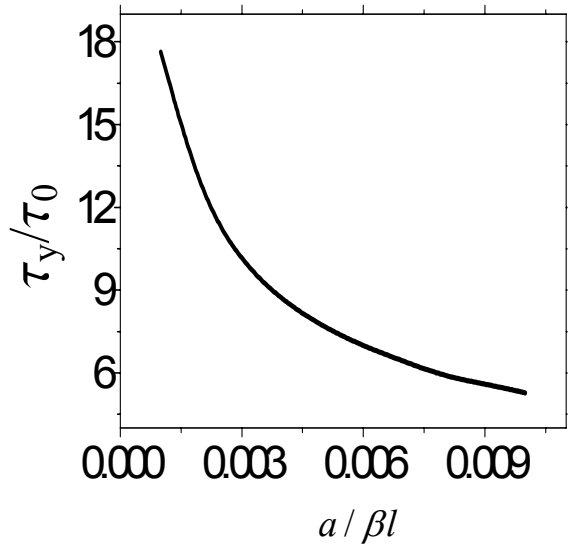

Fig. 5. The stress at the crack tip versus $a /(\beta l)$ for $l=1.0$.

\section{Conclusion}

In this paper, the traditional concepts of the non-local theory are extended to solve the mode-I fracture problem of orthotropic composite materials. Different from the classical elasticity solution, it is found that no stress singularities are present at the crack tips. This enables us to employ the maximum stress criterion to predict fracture in a more natural way for orthotropic composite materials.

\section{References:}

[1] Rubio-Gonzalez, C. Mason, J.J.: Response of finite cracks in orthotropic materials due to concentrated impact shear loads. Journal of Applied Mechanics, 66, 485-491 (1999).

[2] Shol, C.W., Lee, K.Y.: Dynamic response of subsurface interface crack in multi-layered orthotropic half-space under anti-plane shear impact loading. International Journal of Solids and Structures, 38, 3563-3574 (2001).

[3] Kassir, M.K., Bandyopadhyay, K.K.: Impact response of a cracked orthotropic medium. Journal of Applied Mechanics, 50, 630-636 (1983).

[4] Rice, J.R.: A path independent integral and the approximate analysis of strain concentrations by notches and cracks. Journal of Applied Mechanics, 35, 379-386 (1968).

[5] Edelen, D.G.B.: Non-local field theory. In: A.C. Eringen (ed.), Continuum Physics. Vol.4. New York: Academic Press, 75-204 (1976).

[6] Eringen, A.C.: Non-local polar field theory. In: A.C. Eringen (ed.), Continuum Physics. Vol.4. New York: Academic Press, 205-267 (1976).

[7] Green, A.E., Rivilin, R.S.: Multipolar continuum mechanics: functional theory. I. Proceeding of The Royal Society of London A 284, 303-315 (1965).

[8] Pan, K.L., Takeda, N.: Non-local stress field of interface dislocations. Archive of Applied Mechanics. 68, 179-184 (1998).

[9] Eringen, A.C., Speziale, C.G., Kim, B.S.: Crack tip problem in non-local elasticity. Journal of Mechanics and Physics of Solids, 25, 339 (1977). 
[10] Eringen, A.C.: Linear crack subject to shear. International Journal of Fracture, 14, 367-379 (1978).

[11] Eringen, A.C.: Linear crack subject to anti-plane shear. Engineering Fracture Mechanics, 12, 211-219 (1979).

[12] Zhou, Z.G., Han, J.C., Du, S.Y.: Investigation of a Griffith crack subject to anti-plane shear by using the non-local theory. International Journal of Solids and Structures, 36, 3891-3901 (1999).

[13] Zhou, Z.G., Bai, Y.Y., Zhang, X.W.: Scattering of harmonic shear waves by a finite crack by using the non-local theory. International Journal of Engineering Science, 37(5), 609-620 (1999).

[14] Zhou, Z.G.; Du, S.Y. and Wang, B.: Investigation of anti-plane shear behavior of a Griffith crack in a piezoelectric material by using the non-local theory. International Journal of Fracture, 111(2), 105-117 (2001).

[15] Morse, P.M.; Feshbach,H.: Methods of Theoretical Physics, Vol.1, p.926, McGraw-Hill, New York (1958).

[16] Eringen, A.C., Kim, B.S.: Relation between non-local elasticity and lattice dynamics. Crystal Lattice Defects. 7, 51-57 (1977).

[17] Gradshteyn, I.S., Ryzhik, I.M.: Table of integrals, Series and Products. Academic Press, New York (1980).

[18] Erdelyi, A. Tables of Integral Transforms. Vol.1, McGraw-Hill, New York (1954).

[19] Zhou, Z.G. and Wang, B.:Dynamic behavior of two collinear anti-plane shear cracks in a piezoelectric layer bonded to dissimilar half spaces. JSME International Journal, 45(4): 620-628(2002).

[20] Zhou, Z.G., Wang, B. and Yang, L.J.: Investigation of the behavior of an interface crack between two half-planes of orthotropic functionally graded materials by using a new method, JSME International Journal, 47(3): 467-478(2004).

[21] Eringen, A.C.: Interaction of a dislocation with a crack. Journal of Applied Physics, 54, 6811-6817 (1983). 